ARTICLE

\title{
Room temperature high-fidelity holonomic single-qubit gate on a solid-state spin
}

\author{
Silvia Arroyo-Camejo ${ }^{1}$, Andrii Lazariev², Stefan W. Hell ${ }^{1} \&$ Gopalakrishnan Balasubramanian²
}

At its most fundamental level, circuit-based quantum computation relies on the application of controlled phase shift operations on quantum registers. While these operations are generally compromised by noise and imperfections, quantum gates based on geometric phase shifts can provide intrinsically fault-tolerant quantum computing. Here we demonstrate the highfidelity realization of a recently proposed fast (non-adiabatic) and universal (non-Abelian) holonomic single-qubit gate, using an individual solid-state spin qubit under ambient conditions. This fault-tolerant quantum gate provides an elegant means for achieving the fidelity threshold indispensable for implementing quantum error correction protocols. Since we employ a spin qubit associated with a nitrogen-vacancy colour centre in diamond, this system is based on integrable and scalable hardware exhibiting strong analogy to current silicon technology. This quantum gate realization is a promising step towards viable, fault-tolerant quantum computing under ambient conditions.

\footnotetext{
${ }^{1}$ Department of NanoBiophotonics, Max Planck Institute for Biophysical Chemistry, Göttingen, Germany. ${ }^{2}$ Max Planck Research Group Nanoscale Spin Imaging, Max Planck Institute for Biophysical Chemistry, Göttingen, Germany. Correspondence and requests for materials should be addressed to S.A.C. (email: sarroyo@mpibpc.mpg.de) or to S.W.H. (email: shell@mpibpc.mpg.de).
} 
Q uantum gates based on geometric phase shifts $^{1}$ are a promising resource for intrinsically fault-tolerant quantum computing ${ }^{2}$. While initial proposals for geometric quantum computing ${ }^{2}$ based on non-Abelian, adiabatic holonomies ${ }^{3}$ entailed slow gate speeds and were thus prone to decoherence, fast schemes ${ }^{4}$ employing Abelian, nonadiabatic phases ${ }^{5}$ lacked computational universality. Recently, a scheme for non-adiabatic, non-Abelian holonomic quantum computation has been proposed ${ }^{6}$. While the non-Abelian nature of this gate provides computational universality ${ }^{2,7}$, the fact that it is non-adiabatic allows for fast quantum gate operation, high clock frequencies and thus intrinsic protection against decoherence. The superior robustness of this quantum gate against decay, dephasing and parametric imperfections (as for example absolute and relative detuning and pulse errors) has been confirmed analytically and by comprehensive numerical simulations ${ }^{6,8}$. Originally, the proposal for this holonomic quantum gate ${ }^{6}$ was given for a three-level $\Lambda$-type system, where the computational basis states are the mutually uncoupled two lower states, while the upper state acts as an ancilla and remains unpopulated before and after the gate operation. A single-qubit gate based on this scheme ${ }^{6}$ was recently demonstrated on a transmon qubit ${ }^{9}$, but this approach is limited to cryogenic temperatures; another holonomic quantum computing realization on a room temperature liquid nuclear magnetic resonance system ${ }^{10}$ is fundamentally challenged by difficulties to initialize nuclear magnetic resonance systems into pure states.

Here we demonstrate a high-fidelity realization of this holonomic single-qubit gate using an individual solid-state spin qubit under ambient conditions. By selecting the electron spin of a nitrogen-vacancy (NV) centre in diamond to create holonomic quantum gates at room temperature, we choose a quantum computing architecture that is based on integrable $e^{11}$ and scalable $^{12}$ solid-state hardware exhibiting strong analogy to current state-of-the-art silicon technology. In our demonstration we achieve close to perfect process fidelities that comply with the error threshold requirement for the implementation of certain fault-tolerant quantum computing protocols ${ }^{13}$. This realization is a promising step towards viable quantum computing at room temperature.

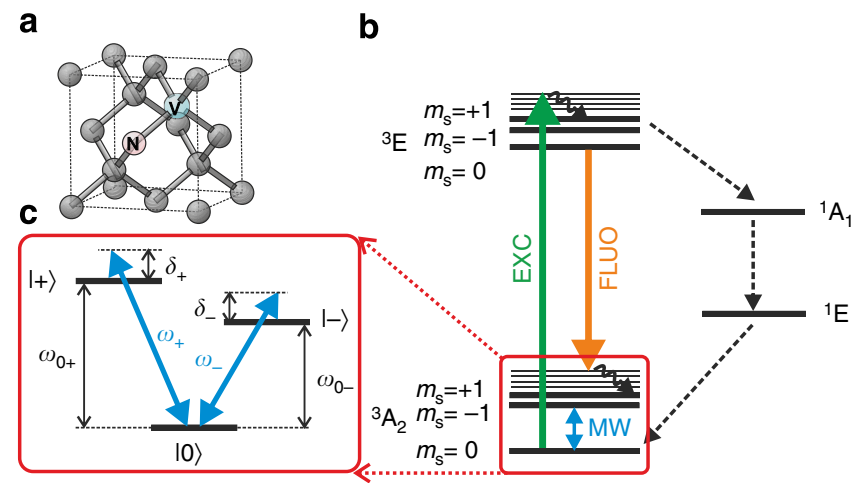

Figure 1 | NV centre geometry and level structure. (a) Illustration of the unit cell of diamond including an NV colour centre. Spin projections $m_{\mathrm{s}}=0, \pm 1$ are defined with respect to the NV symmetry axis. (b) Energy levels of the triplet (left) and singlet states (right) of the NV centre and the applied excitation (EXC) and microwave (MW) fields and the detected fluorescence (FLUO). (c) Three-level V-scheme of the NV ground state triplet employed for the realization of the single-qubit holonomic quantum gate.

\section{Results}

NV centre in diamond. The negatively charged NV centre in diamond holds great promise for quantum sensing ${ }^{14-16}$ and quantum information applications ${ }^{17,18}$. NV electron spins stand out as potential room temperature qubits, because they can be manipulated on the nanosecond time scale ${ }^{19}$ while featuring coherence times of milliseconds under ambient conditions ${ }^{20}$. Both initialization $^{21,22}$ and read-out ${ }^{18}$ of the $\mathrm{NV}$ electron spin, as well as dynamic single-qubit gates ${ }^{23}$ and dynamic entanglement generation of two coupled NV centres ${ }^{24}$ have been demonstrated. First implementations of quantum algorithms were reported recently ${ }^{25}$. As the NV centre fulfils the DiVincenzo criteria for quantum computers remarkably well, the realization of an $\mathrm{NV}$ centre based quantum computer ultimately depends on the achievable fidelities of elementary quantum gates. While quantum error correction codes have been proposed ${ }^{26}$, they will be applicable only if the fidelity of each elementary gate exceeds a certain threshold. Reaching this threshold ${ }^{13}$, (single gate error rates are predicted to range between $10^{-6}$ and $10^{-2}$ ) requires resorting to a hardware that is intrinsically fault-tolerant. To this end, hardware based on geometric phases and decoherence-free subspaces are particularly promising. For negligible hyperfine coupling and strain, the Hamiltonian of the ground state triplet of the NV centre

$$
H / \hbar=D S_{\mathrm{z}}^{2}-\gamma_{\mathrm{e}} \mathbf{B} \cdot \mathbf{S}
$$

is defined by the zero-field splitting $D=2 \pi \times 2.87 \mathrm{GHz}$ and the Zeeman splitting with the external magnetic field $\mathbf{B}$, electron gyromagnetic ratio $\gamma_{\mathrm{e}}$ and the electron spin $\mathbf{S}$. The NV electron spin forms a $\mathrm{V}$-type three-level scheme (cf. Fig. 1c) made up of the $m_{\mathrm{s}}=0$ and $m_{\mathrm{s}}= \pm 1$ levels (denoted by $|0\rangle,|+\rangle$ and $|-\rangle$ in the following). At an external magnetic field of $|\mathbf{B}| \approx 404 \mathrm{G}$, the transitions $|0\rangle \leftrightarrow|+\rangle$ at $\omega_{0+}=2 \pi \times 4.003 \mathrm{GHz}$ and $|0\rangle \leftrightarrow$ $|-\rangle$ at $\omega_{0-}=2 \pi \times 1.738 \mathrm{GHz}$ can be driven coherently by microwave radiation at frequencies $\omega_{+}=\omega_{0+}+\delta_{+}$and $\omega_{-}=\omega_{0-}+\delta_{-}$, respectively. Figure $1 \mathrm{~b}$ displays the NV level scheme indicating the optical transitions used for spin initialization and readout along with the applied microwave field transitions.

Holonomic single-qubit quantum gate. The holonomic quantum gate ${ }^{6}$ is defined on a three-dimensional Hilbert space $\mathcal{H}^{3}$. The computational basis states, here $|+\rangle$ and $|-\rangle$, span a twodimensional subspace $\mathcal{H}^{2}$ of that total space. The holonomic quantum gate is carried out by a transformation of the subspace $\mathcal{H}^{2}$ along a smooth, closed loop $\mathcal{C}:[0, T] \ni t \mapsto \mathcal{H}^{2}(t)$, such that $\mathcal{H}^{2}(0)=\mathcal{H}^{2}(T)$ (see illustration in Fig. 2a). For the duration of the holonomic transformation, the $|0\rangle$ state acts as an idle ancilla that stays unoccupied before and after the quantum gate acts. In the rotating frame the $\mathrm{NV}$ interaction Hamiltonian reads

$$
H_{\text {int }}(t)=\frac{\hbar \Omega(t)}{2}\left(\gamma_{+}|0\rangle\left\langle+\left|+\gamma_{-}\right| 0\right\rangle\langle-|+\text { h.c. }\right),
$$

where the holonomic transformation is carried out through the application of two resonant $\left(\delta_{+}=\delta_{-}=0\right)$ controlled microwave pulses simultaneously driving the $|0\rangle \leftrightarrow|+\rangle$ and $|0\rangle \leftrightarrow|-\rangle$ spin transitions with Rabi frequencies $\Omega(t) \gamma_{+}$and $\Omega(t) \gamma_{-}$, respectively and featuring a joint pulse envelope $\Omega(t)$. To provide a cyclic state evolution, the transformation needs to satisfy $\int_{0}^{T} \Omega(t) d t=2 \pi$ and the Rabi weights $\gamma_{+}$and $\gamma_{-}$need to be properly normalized: $\left|\gamma_{+}\right|+\left|\gamma_{-}\right|=1$. To ensure the parallel transport condition for a purely geometric evolution $\left\langle\psi_{k}\left|H_{\text {int }}\right| \psi_{j}\right\rangle=\delta_{j k}$ the ratio of the Rabi frequencies $\frac{\gamma_{+}}{\gamma}$ has to be kept constant. In this case the dressed system undergoes a Rabi oscillation between the bright state $|b\rangle=-\gamma_{+}^{*}|+\rangle-\gamma_{-}^{*}|-\rangle$ ) and the excited state $|e\rangle=|0\rangle$, whereas the dark state $\left.|d\rangle=-\gamma_{-}|+\rangle+\gamma_{+}|-\rangle\right)$is decoupled from the dynamics. 
a

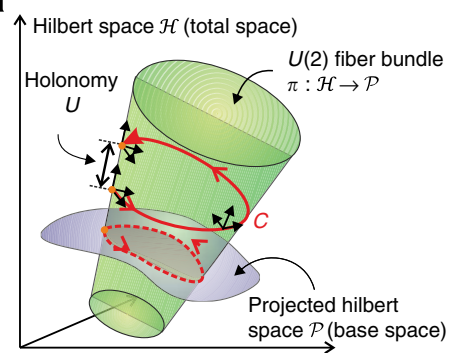

b

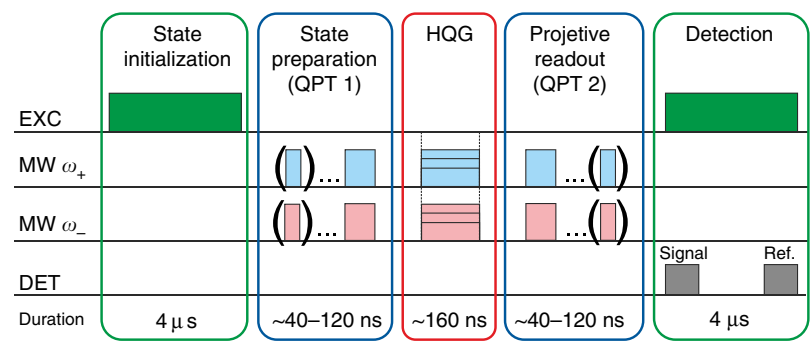

Figure 2 | Concept for the realization of an NV-based holonomic single-qubit gate. (a) Schematic representation of the total Hilbert space and a projected Hilbert space $\mathcal{P}$ therein defined through a projective map $\pi: \mathcal{H} \mapsto \mathcal{P}$ by means of the local $U(2)$ fibre bundle. Driving the system on a suitable loop $\mathcal{C}$ (red curve) on this $U(2)$ fibre bundle of nontrivial topology gives rise to a holonomy. Here, this holonomy is employed for the generation of a relative phase for quantum computation applications. (b) Experimental sequence for the quantum process tomography (QPT): First the NV state is initialized by a green laser pulse (EXC) to state $|0\rangle$. In the following first part of the QPT microwave pulses (MW) tuned to $\omega_{+}$and $\omega_{-}$prepare the spin into one of the nine QPT states $\left|\psi_{j}\right\rangle$. After application of the holonomic quantum gate (HQG), the second part of the QPT project the NV state onto one of the nine measurement bases $\left|\psi_{j}\right\rangle$ by means of microwave pulses (MW). State selective detection concludes the sequence composed of simultaneous application of a green laser pulse (EXC) and signal and reference windows detection (DET). Due to the different numbers and types of pulses necessary for the 81 preparation and readout combinations of the QPT, the durations of the QPT blocks 1 and 2 vary for each QPT measurement.

Table 1 | Theoretical and experimental parameter settings.

\begin{tabular}{|c|c|c|c|c|c|c|}
\hline Quantum gate & Symbolic operator & Evolution operator $\boldsymbol{U}_{+-}$ & Loop parameter $\phi$ & Loop parameter $\theta$ & Rabi weight $\gamma_{+}$ & Rabi weight $\gamma_{-}$ \\
\hline Identity & $I_{+}+I_{-}$ & $\left(\begin{array}{ll}1 & 0 \\
0 & 1\end{array}\right)$ & - & - & - & - \\
\hline Pauli-X & $\sigma_{ \pm}^{\times}$ & $\left(\begin{array}{ll}0 & 1 \\
1 & 0\end{array}\right)$ & $\pi$ & $-\pi / 2$ & $1 / \sqrt{2}$ & $1 / \sqrt{2}$ \\
\hline Pauli-Y & $\sigma_{ \pm}^{\mathrm{y}}$ & $\left(\begin{array}{cc}0 & -i \\
i & 0\end{array}\right)$ & $-\pi / 2$ & $\pi / 2$ & $-i / \sqrt{2}$ & $-1 / \sqrt{2}$ \\
\hline Pauli-Z & $\sigma_{ \pm}^{z}$ & $\left(\begin{array}{cc}1 & 0 \\
0 & -1\end{array}\right)$ & 0 & $\pi$ & 1 & 0 \\
\hline Hadamard & $\frac{\left(\sigma_{ \pm}^{x}+\sigma_{ \pm}^{2}\right)}{\sqrt{2}}$ & $\left(\begin{array}{cc}1 & 1 \\
1 & -1\end{array}\right) / \sqrt{2}$ & 0 & $-\pi / 4$ & $-\cos (\pi / 8)$ & $-\sin (\pi / 8)$ \\
\hline
\end{tabular}

In compliance with the aforementioned conditions, the characteristic structure of the Hamiltonian $H_{\text {int }}(t)$ gives rise to a nontrivial topology of the subspace $\mathcal{H}^{2}$ in the total space $\mathcal{H}^{3}$ and drives the holonomic transformation of the computational states

$$
U_{(+-)}=\left(\begin{array}{cc}
\cos \theta & e^{-i \phi} \sin \theta \\
e^{i \phi} \sin \theta & -\cos \theta
\end{array}\right),
$$

where the Rabi frequency weights can be parameterized by two angles $\theta$ and $\phi$ as $\gamma_{+}=e^{i \phi} \sin (\theta / 2)$ and $\gamma_{-}=-\cos (\theta / 2)$. Hence, the complex valued Rabi frequency ratio $\gamma_{+} / \gamma_{-}$determines the kind of quantum gate by choosing a specific set of $\theta$ and $\phi$ defining a characteristic path $\mathcal{C}(\theta, \phi)$ on the Grassmannian manifold (3;2). In Table 1 , we list the respective parameter sets $(\theta$, $\left.\phi, \gamma_{+} \gamma_{-}\right)$defining some elementary single-qubit quantum gates: the Pauli-X (bit flip gate), Pauli-Y (bit + phase flip gate), Pauli-Z (phase flip gate) and the Hadamard gate. We determine the fidelity of the conducted holonomic quantum gates by performing standard quantum process tomography (QPT) ${ }^{27}$. Figure 3 displays the real parts of the experimentally achieved quantum gate process matrices (coloured bars; imaginary parts are smaller than the respective errors) versus the ideal quantum gates (hollow frame bars). For all three Pauli gates and the Hadamard gate, we achieved total process fidelities around $F=0.98$ (see Table 2) when we apply standard QPT. Note that the QPT, that is, the state preparation before and projective readout after the quantum gate, represents the major part of the experimental sequence (see Fig. 2b). As it is performed through microwave-induced dynamical phase shifts, the QPT is more prone to noise, parameter imperfection and decoherence effects than our holonomic quantum gate. Since the QPT is not part of the quantum gate, it is instructive to assess the corrected fidelities $\tilde{F}_{i}=F_{i} / F_{\text {Id }}$ of the $i$-th holonomic quantum gate after gauging with the fidelity $F_{\text {Id }}$ of a QPT executed without quantum gate (that is, with that of the identity operation). Thereby, we obtain fidelities of the holonomic quantum gates around $\tilde{F}=1.00$ (see Table 2), thus entering the fidelity regime where advanced quantum error correction techniques can become efficacious. Note, that the fidelities reported here were achieved without any of the commonly used post-selection ${ }^{28}$ which, if applied, could increase the fidelity of the demonstrated holonomic quantum gates even further.

\section{Discussion}

Altogether, we showed that NV centre electron spins in diamond enable the realization of high-fidelity, non-adiabatic, non-Abelian holonomic single-qubit gates at room temperature. Acting on the $\mathrm{NV}$ electron spin, this quantum gate is directly compatible with recently developed techniques employing coupling to proximal nuclear spins (such as those provided by ${ }^{13} \mathrm{C}$ nuclei or the NV's own ${ }^{14} \mathrm{~N} /{ }^{15} \mathrm{~N}$ spin), for example, for temporary qubit storage (quantum memory). However, contrary to other demonstrations employing the NV electron spin primarily as a mediator for the control of randomly distributed proximal nuclear spin qubits, our technique allows for the controlled design of a truly engineered 

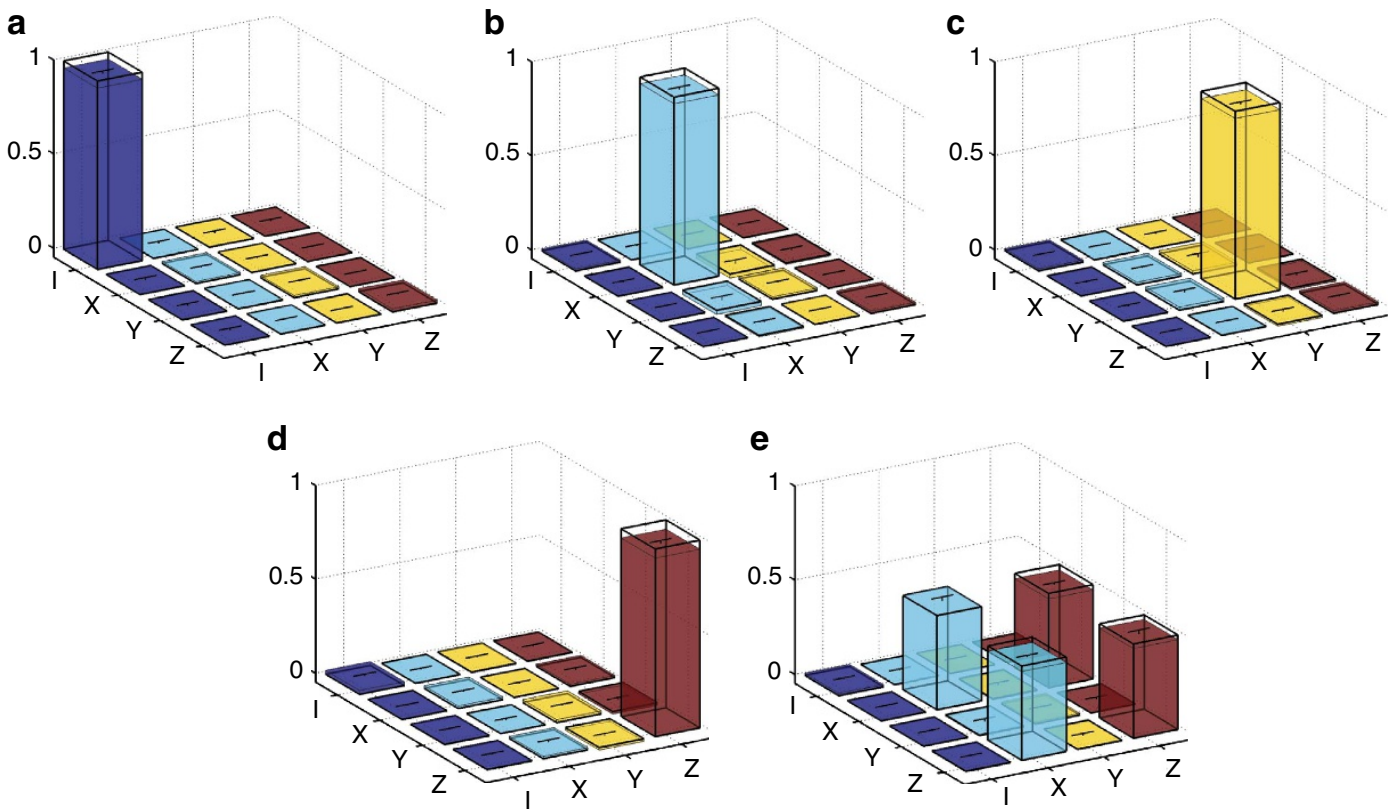

Figure 3 | Representations of the experimentally accomplished quantum processes. (a) Process matrix of the identity operation, (b) the Pauli- $X$, (c) the Pauli-Y, (d) the Pauli-Z and (e) the Hadamard gate (coloured bars: experimental data and and error bars, transparent boxes: ideal quantum gates).

Table 2 | Experimentally achieved fidelities.

\begin{tabular}{lccccc} 
& Identity (no HQG) & Pauli-X & Pauli-Y & Pauli-Z & Hadamard \\
\hline Total fidelity $F_{i}$ & $0.980_{-0.023}^{+0.006}$ & $0.980_{-0.020}^{+0.009}$ & $0.980_{-0.023}^{+0.006}$ & $0.975_{-0.030}^{+0.008}$ & $0.978_{-0.022}^{+0.006}$ \\
HQG fidelity $\tilde{F}_{i}$ & $1.000_{-0.033}^{+0.024}$ & $1.000_{-0.031}^{+0.025}$ & $0.999_{-0.033}^{+0.024}$ & $0.994_{-0.039}^{+0.025}$ & $0.997_{-0.032}^{+0.024}$ \\
\hline
\end{tabular}

QPT, quantum process tomography.

The fidelities of the total experimental sequence (including QPT) $F_{i}=\operatorname{tr}\left(\chi_{\exp } \chi_{\text {theo }}\right)$ and the corresponding fidelities of the $i$-th holonomic quantum gate alone $\tilde{F}_{i}=\frac{F_{i}}{F_{i}}$ obtained from a normalization of the $i$-th total fidelity $F_{\mathrm{i}}$ with respect to the fidelity of the QPT alone $F_{\mathrm{ld}}$ (that is, a QPT run without application of a holonomic quantum gate). The errors denote the $68.3 \%$ confidence intervals of a

Monte-Carlo based error estimation providing an upper bound for the fidelity errors (for details see Supplementary Note 5).

quantum computing architecture based on NV electron spins as computational nodes and employing the built-in NV nitrogen nucleus as a quantum memory.

For universal holonomic quantum computation, the singlequbit gate presented here needs to be combined with a holonomic two-qubit gate. To this end, it is straightforward to consider two electron spins of a pair of adjacent NV centres that are coupled by magnetic dipole-dipole interaction. The far-field optical addressing and readout of individual NV spins could be achieved using the diffraction-unlimited resolution provided by stimulated emission depletion microscopy ${ }^{29}$, which is our long-term vision. In combination with optimal control techniques ${ }^{30}$ such a universal set of holonomic quantum gates can allow for a truly scalable and fault-tolerant quantum computation architecture based on room temperature solid-state hardware. Moreover, the demonstrated robust high-fidelity holonomic phase gates could be employed for other challenging spin manipulation applications to enhance high-fidelity dynamical decoupling protocols or to allow for robust high-sensitivity quantum sensing.

\section{Methods}

Electron spin manipulation. The gate was realized on a naturally occurring NV centre in synthetically grown, electronic grade diamond. For spin initialization and fluorescence readout, the NV centres were optically addressed by a $532 \mathrm{~nm}$ continuous wave laser via a home-build confocal microscope. The microwave pulses were synthesized by a Tektronix AWG 7122C, amplified and coupled into a microwave strip line spanned across the diamond sample. Typically the Rabi frequencies were around $6 \mathrm{MHz}$. The external magnetic field was applied through a permanent magnet and aligned to be parallel to the NV axis to an angle deviation of $\left\langle 2^{\circ}\right.$. The lifetimes of the $\left.\mid+\right\rangle$ and $|-\rangle$ states with respect to the $|0\rangle$ were both $T_{1}=(5 \pm 1) \mathrm{ms}$, while the coherence times of the $|+\rangle$ and $|-\rangle$ states with respect to the $|0\rangle$ state were both $T_{2}^{*}=(7 \pm 1.0) \mu$ s. By applying a magnetic field of $|\mathbf{B}| \approx 404 \mathrm{G}$, we tuned the NV system near the triplet excited state level anti-crossing, providing us with a polarization of the ${ }^{14} \mathrm{~N}$ nuclear spin into the $m_{I}=+1$ hyperfine state.

Quantum process tomography. For the evaluation of the experimentally achieved quantum gates, we conducted standard QPT ${ }^{27}$. An arbitrary operation $\epsilon$ acting on an initial state $\rho^{\text {in }}$ and generating the final state $\rho^{\text {out }}$ can be described by a quantum process matrix $\chi_{m n}$ in terms of a quantum dynamical map $\rho^{\text {out }}=\mathcal{E}\left(\rho^{\text {in }}\right)=\sum_{m n} \chi_{m n} E_{m} \rho^{\text {in }} E_{n}^{\dagger}$, where the $E_{m} \in S U(d)$ represent a full set of orthogonal basis operators (here, the Gell-Mann operators as the generators of the $S U(3))$. The fidelity of the quantum process is then given by the overlap between the experimental and theoretical representation of the process matrix $\chi_{\exp }$ and $\chi_{\text {theor }}$ respectively: $F=\operatorname{tr}\left(\chi_{\exp } \cdot \chi_{\text {theo }}\right)$. Here, the QPT is performed in 81 runs, in each of which the system has to be initialized into one of nine (quasi-)pure states $\left|\psi_{j}\right\rangle \in\left(\left|\psi_{1}\right\rangle,\left|\psi_{2}\right\rangle, \ldots,\left|\psi_{9}\right\rangle\right)$, where the $\left|\psi_{j}\right\rangle$ are chosen such that the corresponding density matrices $\rho_{j}=\left|\psi_{j}\right\rangle\left\langle\psi_{j}\right|$ form a basis set for the space of matrices: $\rho=\sum_{j} q_{j}\left|\psi_{j}\right\rangle\left\langle\psi_{j}\right|$. The reconstructed process matrix $\chi_{m n}$ was fitted by means of a maximum-likelihood estimation procedure assuming the process matrix to be completely positive and not trace increasing (for details see Supplementary Notes 1-5).

\section{References}

1. Berry, M. V. Quantal phase factors accompanying adiabatic changes. Proc. $R$. Soc. Lond. A 392, 45-57 (1984).

2. Zanardi, P. \& Rasetti, M. Holonomic quantum computation. Phys. Lett. A 264, 94-99 (1999).

3. Wilczek, F. \& Zee, A. Appearance of gauge structure in simple dynamical systems. Phys. Rev. Lett. 52, 2111-2114 (1984). 
4. Xiang-Bin, W. \& Keiji, M. Nonadiabatic conditional geometric phase shift with NMR. Phys. Rev. Lett. 87, 097901 (2001).

5. Aharonov, Y. \& Anandan, J. Phase change during a cyclic quantum evolution. Phys. Rev. Lett. 58, 1593-1596 (1987).

6. Sjöqvist, E. et al. Non-adiabatic holonomic quantum computation. New. J. Phys. 14, 103035 (2012)

7. Anandan, J. Non-adiabatic non-abelian geometric phase. Phys. Lett. A 133, 171-175 (1988).

8. Johansson, M. et al. Robustness of nonadiabatic holonomic gates. Phys. Rev. A 86, 062322 (2012).

9. Abdumalikov, A. A. et al. Experimental realization of non-Abelian non-adiabatic geometric gates. Nature 496, 482-485 (2013).

10. Feng, G., Xu, G. \& Long, G. Experimental realization of nonadiabatic holonomic quantum computation. Phys. Rev. Lett. 110, 190501 (2013).

11. Toyli, D. M., Weis, C. D., Fuchs, G. D., Schenkel, T. \& Awschalom, D. D. Chip-scale nanofabrication of single spins and spin arrays in diamond. Nano Lett. 10, 3168-3172 (2010).

12. Yao, N. Y. et al. Scalable architecture for a room temperature solid-state quantum information processor. Nat. Commun. 3, 800 (2012).

13. Knill, E. Quantum computing with realistically noisy devices. Nature 434, 39-44 (2005).

14. Balasubramanian, G. et al. Nanoscale imaging magnetometry with diamond spins under ambient conditions. Nature 455, 648-651 (2008).

15. Maze, J. R. et al. Nanoscale magnetic sensing with an individual electronic spin in diamond. Nature 455, 644-647 (2008).

16. Dolde, F. et al. Electric-field sensing using single diamond spins. Nat. Physics 7, 459-463 (2011)

17. Wrachtrup, J. \& Jelezko, F. Processing quantum information in diamond. J. Phys. Condens. Matter 18, S807-S824 (2006).

18. Dutt, M. V. G. et al. Quantum register based on individual electronic and nuclear spin qubits in diamond. Science 316, 1312-1316 (2007).

19. Fuchs, G. D., Dobrovitski, V. V., Toyli, D. M., Heremans, F. J. \& Awschalom, D. D. Gigahertz dynamics of a strongly driven single quantum spin. Science 326, 1520-1522 (2009).

20. Bar-Gill, N., Pham, L. M., Jarmola, A., Budker, D. \& Walsworth, R. L. Solid-state electronic spin coherence time approaching one second. Nat. Commun. 4, 1743 (2013).

21. Loubser, J. H. N. \& van Wyk, J. A. Electron spin resonance in annealed type 1b diamond. Diamond Res. 11, 4-7 (1977).

22. Gaebel, T. et al. Room-temperature coherent coupling of single spins in diamond. Nat. Physics 2, 408-413 (2006).

23. Kennedy, T. A. et al. Single-qubit operations with the nitrogen-vacancy center in diamond. Phys. Status Solidi B 233, 416-426 (2002).

24. Dolde, F. et al. Room-temperature entanglement between single defect spins in diamond. Nat. Physics 9, 139-143 (2013).
25. van der Sar, T. et al. Decoherence-protected quantum gates for a hybrid solidstate spin register. Nature 484, 82-86 (2012).

26. Shor, P. W. Scheme for reducing decoherence in quantum computer memory. Phys. Rev. A 52, R2493-R2496 (1995).

27. O'Brien, J. L. et al. Quantum process tomography of a controlled-NOT gate. Phys. Rev. Lett. 93, 080502 (2004).

28. Waldherr, G. et al. High-dynamic-range magnetometry with a single nuclear spin in diamond. Nat. Nanotechnol. 7, 105-108 (2012).

29. Rittweger, E., Han, K. Y., Irvine, S. E., Eggeling, C. \& Hell, S. W. STED microscopy reveals crystal colour centres with nanometric resolution. Nat. Photonics 3, 144-147 (2009).

30. Dolde, F. et al. High-fidelity spin entanglement using optimal control. Nat. Commun. 5, 3371 (2014).

\section{Acknowledgements}

We thank D. Mesterhazy for fruitful discussions. We also thank Fedor Jelezko and Alex Sushkov for helpful comments on the manuscript. We acknowledge funding from the Volkswagen Stiftung.

\section{Author contributions}

S.A.-C. conceived the experiment, evaluated the data and wrote the manuscript. G.B. and A.L. designed and constructed the setup. S.A.-C. and A.L. performed the experiments. S.W.H. and G.B. supervised the project. All authors contributed with discussions and to the final form of the manuscript.

\section{Additional information}

Supplementary Information accompanies this paper at http://www.nature.com/ naturecommunications

Competing financial interests: The authors declare no competing financial interests

Reprints and permission information is available online at http://npg.nature.com/ reprintsandpermissions/

How to cite this article: Arroyo-Camejo, S. et al. Room temperature high-fidelity holonomic single-qubit gate on a solid-state spin. Nat. Commun. 5:4870 doi: $10.1038 /$ ncomms5870 (2014).

(C) This work is licensed under a Creative Commons AttributionCY NC ND NonCommercial-NoDerivs 4.0 International License. The images or other third party material in this article are included in the article's Creative Commons license, unless indicated otherwise in the credit line; if the material is not included under the Creative Commons license, users will need to obtain permission from the license holder to reproduce the material. To view a copy of this license, visit http:// creativecommons.org/licenses/by-nc-nd/4.0/ 